\title{
Peri-Implant Bone Behavior after Single Drill versus Multiple Sequence for Osteotomy Drill
}

\author{
Sergio Alexandre Gehrke $\left(\mathbb{D},{ }^{1}\right.$ Raphaél Bettach, ${ }^{2,3}$ Jaime Sardá Aramburú Júnior, ${ }^{4}$ \\ Juan Carlos Prados-Frutos $\left(10,{ }^{5}\right.$ Massimo Del Fabbro ${ }^{\oplus},{ }^{6}$ and Jamil Awad Shibli $\oplus^{7}$ \\ ${ }^{1}$ Department of Research, Biotecnos Research Center, Montevideo, Uruguay \\ ${ }^{2}$ New York University, New York, NY, USA \\ ${ }^{3}$ Private Practice, Gretz-Armainvilliers, France \\ ${ }^{4}$ Veterinary Program, Itapiranga Faculty, Itapiranga, SC, Brazil \\ ${ }^{5}$ Department of Medicine and Surgery (Stomatology Area), Rey Juan Carlos University, Madrid, Spain \\ ${ }^{6}$ Research Center in Oral Health, Department of Biomedical, Surgical and Dental Sciences, \\ IRCCS Istituto Ortopedico Galeazzi, Università degli Studi di Milano, Milano, Italy \\ ${ }^{7}$ Department of Periodontology and Oral Implantology, University of Guarulhos, Guarulhos, SP, Brazil
}

Correspondence should be addressed to Sergio Alexandre Gehrke; sergio.gehrke@hotmail.com

Received 23 July 2017; Accepted 7 February 2018; Published 11 April 2018

Academic Editor: Hassan Maghaireh

Copyright (C) 2018 Sergio Alexandre Gehrke et al. This is an open access article distributed under the Creative Commons Attribution License, which permits unrestricted use, distribution, and reproduction in any medium, provided the original work is properly cited.

\begin{abstract}
Objectives. The present study aims to compare the drilling protocol effect on osseointegration event in three commercially available titanium dental implants with different drill protocol using a rabbit tibia model. Materials and Methods. Three different drilling sequences were compared as follows: drilling sequence using a single unique drill of $4.2 \mathrm{~mm}$ conical implant (Group 1), drilling sequence using 3 consecutive cylindrical drills for a $4.1 \mathrm{~mm}$ cylindrical implant (Group 2), and drilling sequence using 3 consecutive conical drills for a $4.3 \mathrm{~mm}$ conical implant (Group 3). For each group, 18 drilling procedures and implant placements were performed, totalizing 54 commercially available titanium dental implants. The samples were removed 6 weeks after implantation. Resonance frequency analyses (RFA) were performed immediately after the implantation, and at 6 weeks removal torque test (RTt) and histological analysis were performed. Results. The RFA measured showed statistical difference between the groups in time 1 and no significant statistical differences in time $2(p>0.05)$. In the RTt no significant difference was found between the 3 groups tested. Histomorphometric analysis showed no significant difference between groups in the bone-to-implant contact $\%(p>0.05)$. Conclusion. In the present preclinical study, osteotomy using a single bur did not show differences regarding the proposed and evaluated tests parameters for assessing the peri-implant behavior.
\end{abstract}

\section{Introduction}

The rehabilitation of tooth loss with dental titanium was documented and shown to have more than $98 \%$ of success rate $[1,2]$. Osseointegration is the first step to the success of this type of treatment, which was defined as a direct contact between the bone tissue and the implant without the presence of fibers (soft tissue) [3]. It has been suggested that success of osseointegration is related to 6 main criteria: material biocompatibility, implant design, surface morphology, conditions of the implanted tissues, surgical technique, and loading conditions [4]. Among these, the excessive surgical trauma, prosthesis overload, misfit of suprastructures, or implanted area with infection can be considered the critical modifiable factors $[5,6]$.

Minimizing surgical trauma to bone tissue during the osteotomy is a controllable factor and may contribute to the osseointegration success [7]. Therefore, while drilling the bone, the temperature control during the osteotomies, due to attrition of burs, can cause tissue alterations and cells death, mainly damage in the organic portion of bone tissue $[8,9]$, may interfere directly in the process of bone healing (osseointegration), and can induce the crestal bone loss and to influence implant survival [10-13]. 
On the other hand, regarding the implanted material, there is still a lack of knowledge about the events related to a bone response in relation to the different surface types and which would be the most appropriate. Some steps of the actual biological events are the initial activation of bone healing around the implants, such as protein adsorption, interaction between cells and implant surface, migration and differentiation of progenitor cells, and tissue formation at the bone-implant interface, supposed to be affected by the surface morphology of the implant and its physicochemical structure [14-17]. In addition, the initial stability of the implant is directly linked to its macro design, such as cylindrical or tapered design, its length and diameter, and the type of turns and the distance between them. All these factors may act positively or negatively on implant locking in bone tissue [18]. In this regard, Gehrke et al. [19], published a study that showed measurements of the insertion torque value (ITV), implant stability quotient (ISQ), and precision of osteotomy using conventional and simplified (a single drilling step is used) drilling systems, and the evaluation showed that the hole quality and the ITV promote a significant increase in the primary stability of the implants. Therefore, the system using a single drill for the osteotomy showed significantly higher ITV and ISQ than the systems tested using a multiple-drill sequence for the osteotomy.

Recently an increasing interest has been shown in the scientific community regarding the investigation of different drills design and osteotomy protocols, its results on the bone trauma control, and consequently its effects on the bone healing [20-23]. However, there is little information available in the literature about what should be the ideal progression for increasing the diameter during osteotomy and/or whether it should be progressive. However, it was assumed that the osteotomy was performed by incremental steps, increasing the drilling diameter slowly and thus minimizing trauma to the bone tissue. There is little evidence in the literature about the effects of different milling protocols and which would be ideal and therefore less traumatic. However, Gehrke [22] showed in a histological study that the use of new drill (discardable drill) can promote better results in comparison with multiple use drills. More recently, Bettach et al. [24] published a human study where it became evident that the use of a drilling protocol with a single drill can present a high success rate of osseointegration of the implants [24]. This type of protocol can bring some important advantages from the point of view of required working time and manipulation over bone tissue (perforation) when compared to traditional staggered protocols, which require obviously longer time steps. Thus, there should be a balance between the precision in the positioning required by the implant in terms of the inclination, diameter, and shape of the osteotomy, thus seeking an ideal anchorage (stability) of the implants and rationing of the total time necessary to perform it. In addition, another proposed care was that the final drilling bur should be shorter; thus, hypothetically, it decreases the exposure for an extended time and, consequently, the possibility of generating more heating to the bone tissue. In this sense, the proposition to investigate the reduction of the number of drills during implant osteotomy, using a single

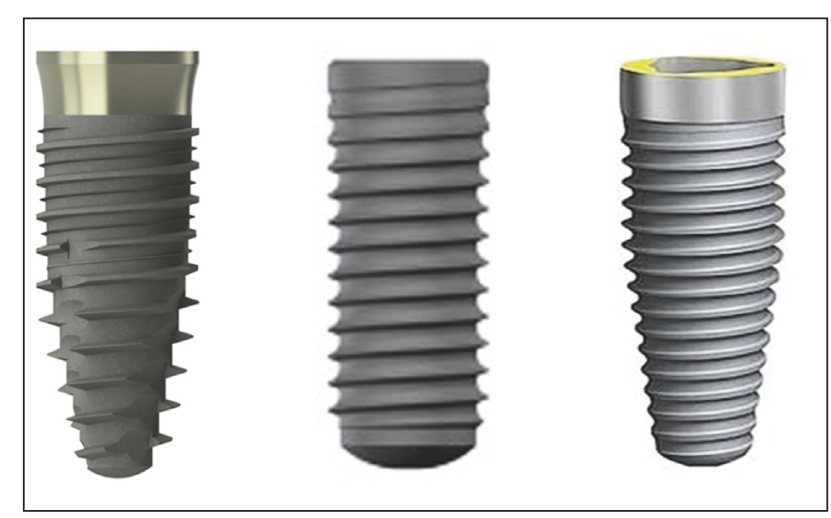

FIGURE 1: Images of the implants used in the study.

drill with high cutting power, was analyzed and compared with the conventional sequences using multiple drills. This new methodology and technology tested aim to provide results similar to the conventional sequence (multiple) used until today [20-23].

The purpose of this study was to compare, through biomechanical and histological analysis, the effects on the osseointegration event using a reduced protocol for osteotomy (only one drill) with the conventional drilling protocol (multiple) for implant osteotomy, using a rabbit tibia model.

The null hypothesis was that the use of a reduced protocol for osteotomy (one drill) did not affect the osseointegration process of the implant when compared to the osseointegration of implants installed using conventional osteotomy (multiple drills).

\section{Materials and Methods}

Fifty-four commercially titanium dental implants with 3 different designs (Figure 1) and drill sequences for osteotomy protocol (Figure 2) were divided into 3 groups $(n=18 \mathrm{im}$ plants per group):

Group 1. One drill for conical implant with $\varnothing 4.2 \mathrm{~mm}$ and $10 \mathrm{~mm}$ in length (Implants Diffusion International, Montreuil, France), with recommended speed in $1500 \mathrm{rpm}$.

Group 2. Sequential drills for cylindrical implant with $\varnothing 4.1 \mathrm{~mm}$ and $8 \mathrm{~mm}$ in length (BoneLevel, Straumann, Basel, Switzerland): Ø2.2 mm (800 rpm), Ø2.8 mm (600 rpm), and $\varnothing 3.5 \mathrm{~mm}$ (500 rpm) [25].

Group 3. Sequential drills for conical implant with $\varnothing 4.3 \mathrm{~mm}$ and $8 \mathrm{~mm}$ in length (NobelReplace ${ }^{\circledR}$ implant, Nobel Biocare, Göteborg, Sweden): Ø2 mm (2000 rpm), Ø3.5 mm (800 rpm), and $\varnothing 4.3 \mathrm{~mm}(800 \mathrm{rpm})$ [26].

In Group 1, the implant surface was prepared by sandblasting acid (SLA) using blasting with aluminum oxide plus acids attack and then a thermic treatment (IDI, Montreuil, France); in Group 2, the implant surface is treated by a SLA procedure with aluminum oxide for blasting plus acids 


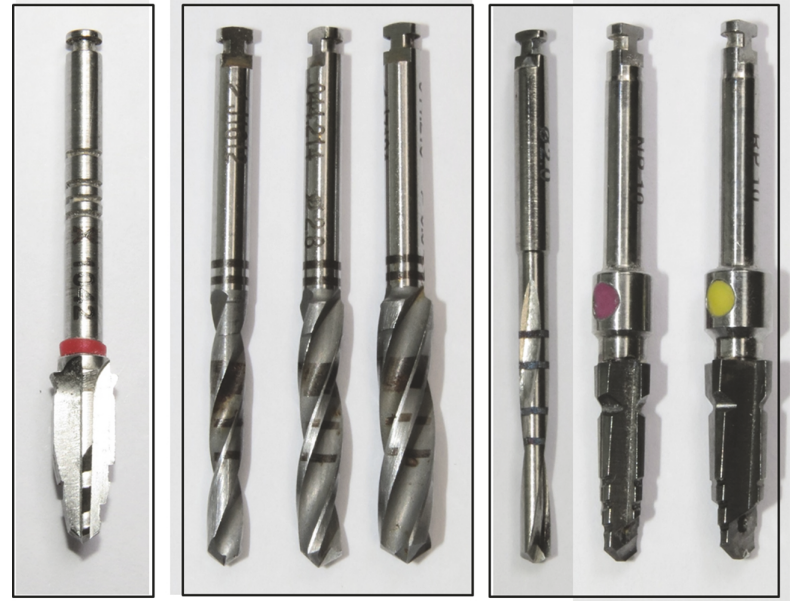

Figure 2: Drill sequences used for osteotomy in the groups 1-3, respectively.

attack (Straumann, Basel, Switzerland); and, in Group 3, the implants were treated by anodization method (TiUnite ${ }^{\circledR}$, Nobel Biocare, Sweden). All implants were purchased from their local resellers in the same conditions under which they are marketed for clinical use.

\subsection{Animals and Surgical Procedure. Nine New Zealand} white adult rabbits weighing between 4 to $4.5 \mathrm{~kg}$ were used for the present preclinical study. This study was approved by the Ethics Committee of the Itapiranga Faculty, Itapiranga, Santa Catarina, Brazil (\#004-09-2015). This type of animal presents adequate conditions for the evaluation of the healing of the bone tissue around implants [27, 28] and is frequently used for experimental preclinical studies [23]. To anesthetize the animals, ketamine $35 \mathrm{mg} / \mathrm{kg}$ intramuscular (Agener Pharmaceutical, Brazil) was used plus Rompun $5 \mathrm{mg} / \mathrm{kg}$ (Bayer, São Paulo, Brazil). Additionally, Acepran $0.75 \mathrm{mg} / \mathrm{kg}$ (Univet, São Paulo, Brazil) was used as tranquilizer. Besides, a local anesthetic (3\% Prilocaine-felypressin, Astra, Mexico) was subcutaneously administered near of the location of implantation to make vasoconstriction and control the pain. Then, a tissue incision was made to access the bone, the flap was lifted exposing the bone tissue, and the perforations were performed under abundant irrigation using the milling sequence determined and previously written for each implant model (each group). One implant of each group was placed in each tibia ( 3 per tibia), with the sites being numbered from proximal to distal as 1-3 (Figure 3), and distributed equally for each group. The implants were anchored bicortically; however the cervical portion of all implants was positioned at the level of the cortical bone. The control of the torque at the level of $20 \pm 5 \mathrm{Ncm}$ during the implant insertion was performed by a manual torquimeter and, finally, the implant stability quotient was measured. The suture using a 5-0 nylon were performed with individual simple points. Postoperatively, an antibiotic dose was administrated (600,000 IU Benzetacil). In the postsurgery, all animals received the care standardized by the veterinary hospital, that is, individual places with

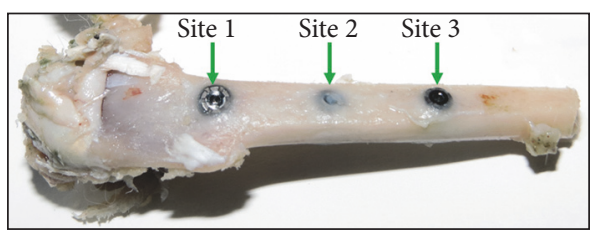

FIgURE 3: Image of the tibia showing the site numeration used to the implants distribution.

12-hour cycles of light/dark, $21^{\circ} \mathrm{C}$ of temperature, and ad libitum diet. The postoperative period of the animals was within normality, that is, without complications or adverse events. Six weeks after the surgery, all were euthanized using an intravenous overdose of the anaesthetics $(2 \mathrm{ml}$ of ketamine plus $1 \mathrm{ml}$ of xylazine). The 2 tibias were removed and packed in bottles with $10 \%$ formalin solution. Then, they were taken to the laboratory (Biotecnos, Santa Maria, Brazil) for immediate analysis.

2.2. Resonance Frequency Analysis. Resonance frequency analysis (RFA) was performed to evaluate the implant stability. A Smartpeg ${ }^{\mathrm{TM}}$ (Integration Diagnostics AB, Göteborg, Sweden) was screwed in the implants with approximately $5 \mathrm{~N}$. The implant stability quotient (ISQ) was measured using the Osstell ${ }^{\mathrm{TM}}$ Mentor (Integration Diagnostics AB, Göteborg, Sweden), with the sensor being positioned at a distance of 2 or $3 \mathrm{~mm}$ from the Smartpeg. The RFA of each implant was measured immediately after the installation and after the sacrifice ( 6 weeks). The ISQ data used for each implant sample was an average of the collected value of 2 directions (proximal to distal and lateral to medial) (Figure 4).

2.3. Removal Torque Test. Nine implants (3 per group) were removed in contra-torque. These implants were removed in site 1 (more proximal site) of the tibia by lot between the groups. A computerized torque testing machine (CME, Técnica Industrial Oswaldo Filizola, São Paulo, Brazil), used in other studies and developed by our group, was used in the present study [29] (Figure 5). The test speed used was $4 \mathrm{rpm}$, the maximum torque values, measured upon initiating the reverse rotation of each implant, were recorded, and the mean torque for each group was calculated.

2.4. Histomorphometric Analysis. Forty-five osseointegrated implants (15 per group) were treated by a dehydration process in sequential alcohols concentration (50 to 100\%) and embedded historesin (Technovit 9100 VLC, Kulzer, Germany). Then, the blocks with the samples were cut into the portion corresponding to the center of each implant in slices of $\sim 50 \mu \mathrm{m}$ thick using a micrometric cutter (Isomet 2000, Buehler, Germany). The histological slides were set, abraded, and polished by a sequence of sticks up to $\sim 30 \mu \mathrm{m}$ thick and finally stained with picrosirius hematoxylin and analyzed histologically.

In each sample the bone tissue surrounding the implant in the cortical bone portion was histologically evaluated, and the percentage of bone-to-implant contact (BIC\%) was made 


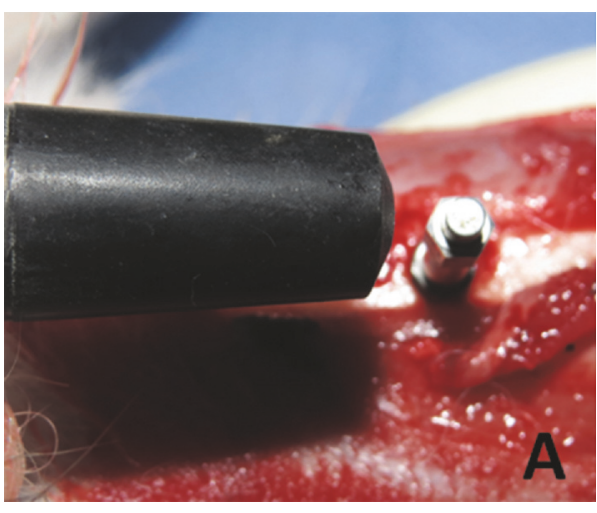

(a)

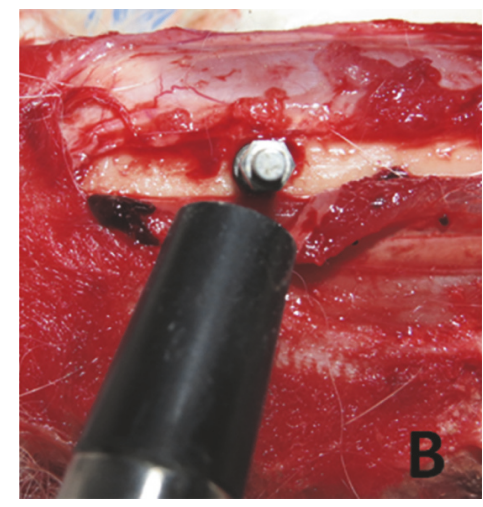

(b)

FIGURE 4: Images showing the 2 directions of the ISQ measurements of each implant. (a) Proximal to distal and (b) lateral to medial.

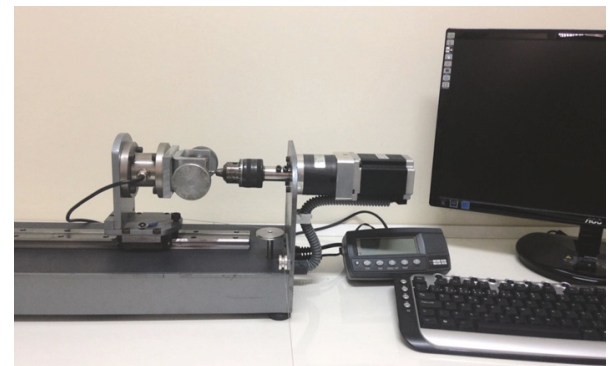

FIGURE 5: Image of the computerized torque machine used in the removal torque test.

using a light microscope (EOS 200, Nikon, Tokyo, Japan). The measurements of BIC\% were performed using the software Image Tool version 5.02 for Microsoft Windows ${ }^{\mathrm{TM}}$ on the digitalized images. BIC\% was calculated as the percentage of bone that was in direct contact with the implant surface.

2.5. Data Analysis. The data were longitudinally compared among the groups with the Friedman test and one-way analysis of variance (ANOVA) test for repeated measures. The Mann-Whitney $U$ test was used for the comparative analysis among the 3 groups in the same test. These statistical analyses were made with the computational program GraphPad Prism 5.01 (GraphPad Software Inc., San Diego, CA, USA). The level of significance was set at $\alpha=0.05$.

\section{Results}

In performed postoperative controls, no healing problem was observed, presenting adequate evolution in the weekly evaluations. After sacrifice (6 weeks), all implants were osseointegrated. Six weeks after the surgical implantation, all implants were osseointegrated.

3.1. Resonance Frequency Analysis (RFA). The measured values and statistical analysis of RFA for the 2 times measured of the 3 groups are shown in Table 1. Performing the statistical test within the times (baseline and 6 weeks) among the groups studied, the values showed statistically significant differences in time $1(p=0.005)$ and no difference in time $2(p=0.068)$. The data distribution in each time of the groups is presented in the graphs of Figure 6.

3.2. Removal Torque Test (RTt). In RTt, all samples presented a good stability in the bone tissue. The mean resistance to removal torque values and standard deviation was $95.7 \pm$ $3.21 \mathrm{~N}$ for Group 1, $91.0 \pm 2.65 \mathrm{~N}$ for Group 2, and $91.0 \pm$ $3.61 \mathrm{~N}$ for Group 3. The statistical test showed no differences between the groups $(p=0.622)$.

3.3. Histological Analysis. Histological observations showed adequate bone organization and mineralization around the implants at 6 weeks in all groups (Figures 7-9). The BIC\% values measured in the cortical bone portion were $71.7 \pm$ $2.94 \%$ for Group 1, $70.8 \pm 2.43 \%$ for Group 2, and $70.8 \pm$ $3.30 \%$ for Group 3. The data analysis did not show statistical differences between the 3 groups $(p=0.644)$.

\section{Discussion}

Recently, a clinical study evaluation of 350 implants installed in several clinical procedures showed excellent results using a single drill system for osteotomy, with $98 \%$ of implant survival [26]. Then, Gehrke et al. [30] investigated the possible relationship between this good result and the bone heat generation during the osteotomy (less surgical trauma) and concluded that this drilling system (using one drill), prepared to perform the osteotomy for implant placement in a single drilling maneuver, did not provide temperature rise in bone tissue compared to drilling systems using a stepped sequence (multiple drills) for osteotomy of the implant bed. In this sense, the present biomechanical and histological investigation in animals was developed to check and conclude if this protocol using a single drill for osteotomy did not change the osseointegration events and so this might be a possible explanation for the success of this technique. 
TABLE 1: Friedman test of ISQ analysis and measurements at baseline (initial) and at 6 weeks. Results as mean and medians. Mann-Whitney $U$ test to compare intragroups $(p<0.05)$.

\begin{tabular}{lccccc}
\hline ISQ value & \multicolumn{2}{c}{ Baseline } & \multicolumn{2}{c}{6 weeks } & Median \\
\hline Group 1 & Mean \pm Sd & Median & Mean \pm Sd & 79 & $<0.0001$ \\
Group 2 & $67.9 \pm 2.69$ & 68 & $78.7 \pm 5.41$ & 77 & $<0.0001$ \\
Group 3 & $66.8 \pm 2.83$ & 66 & $75.1 \pm 5.26$ & 76 & $<0.0001$ \\
$p$ value (intergroup) & $63.8 \pm 4.36$ & 65 & $74.3 \pm 5.18$ & 0.068 & \\
\hline
\end{tabular}
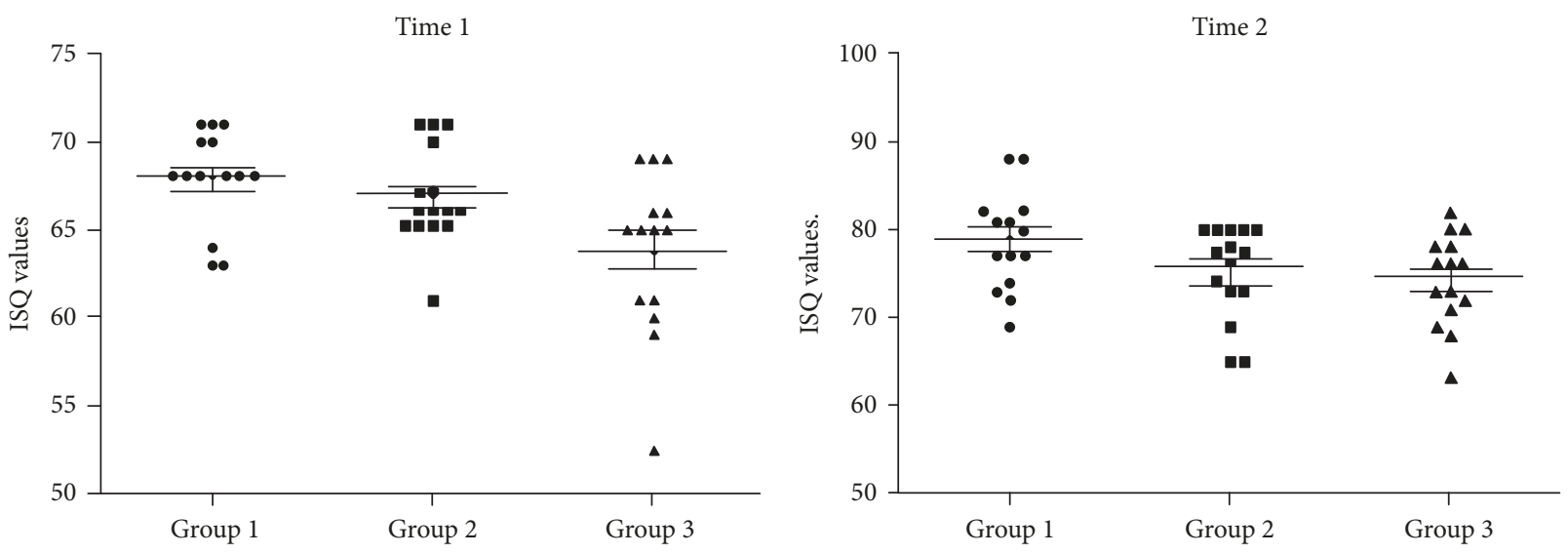

FIGURE 6: Graphs of the ISQ values distribution of the 3 groups in the 2 times (baseline and 6 weeks).

In this histological study, we evaluated the bone response (osseointegration) of three different implant models with different osteotomy drilling systems. The findings showed that the use of only one drill for the osteotomy provided similar biomechanical and histological response than using a conventional multiple drilling. In a histological evaluation, several studies showed that bone tissue behavior in implants placed with a simplified protocol is similar to the conventional protocol using multiple sequential drills [20-23]. However, even if the considerations seem obvious, the differences between the osteotomy protocols should be considered as a possibility of reducing the surgical trauma during the installation of the implants.

Several investigations have studied the effects of macro design of implants with regard to the healing and stability events [31-33]. These modifications in implant macro design at the beginning were proposed to accelerate the osseointegration of the implants increasing the initial stability and later increase the rate of contact between bone and implant after the period of bone healing, which benefits the distribution of the loads generated when these structures enter into a masticatory function [34]. In the present study, three implant designs were used and, although they presented a great variation in their design, the response was quite similar between the groups. Thus, it would be possible to suggest that osteotomy may be the determining factor for obtaining one of the main requirements for the success of osseointegration, the initial stability.

By making a relation between the reaction provided by the type of osteotomy performed and the surgical trauma on the cortical bone tissue, several considerations can be highlighted in order to reduce the possible effects of physical stress. To reduce surgical trauma during the osteotomy procedure, several points should be considered: the structural design of the cutter, such as cutting blade, blade angles, edging, and dimensions; the speed of rotation, the force to be exerted (applied pressure), the amount of irrigation, the maximum torque applied, and the use of sequential diameter drills or a single step $[35,36]$. On the other hand, the variations presented by the locations where the osteotomies will be performed, such as bone tissue volume and density, and consequently the time required for osteotomy execution are factors that may interfere with the trauma generated during surgery for the installation of implants.

Two biomechanical tests, removal torque test (RTt) and resonance frequency analysis (RFA), were used to evaluate the 3 different implant designs used in this study. The RTt serves as a parameter to determine the resistance of the connection between the bone tissue and the implant $[37,38]$, and the high values of resistance in the counter-torque for implant removal indicate that there is a high density of bone tissue and a strong connection between bone and implant [38]. However, the RFA possibility of measuring implant stability at any time during implant treatment, without adding load, can be considered a noninvasive method for this type of evaluation $[39,40]$.

Gehrke and da Silva Neto [41] demonstrated in a clinical human study that the ISQ values have a direct relationship with the bone density (maxilla and mandible), even though the measurements show stability values in short periods, 


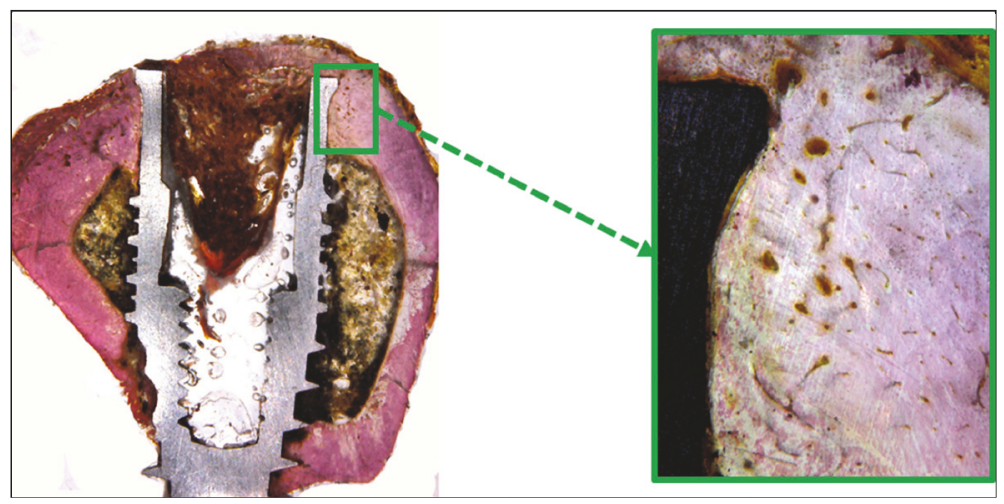

FIGURE 7: Histological pictures showing the bone healing around the implant after 6 weeks of Group 1. Magnification: 4 and 100x, respectively. Picrosirius-hematoxylin staining.

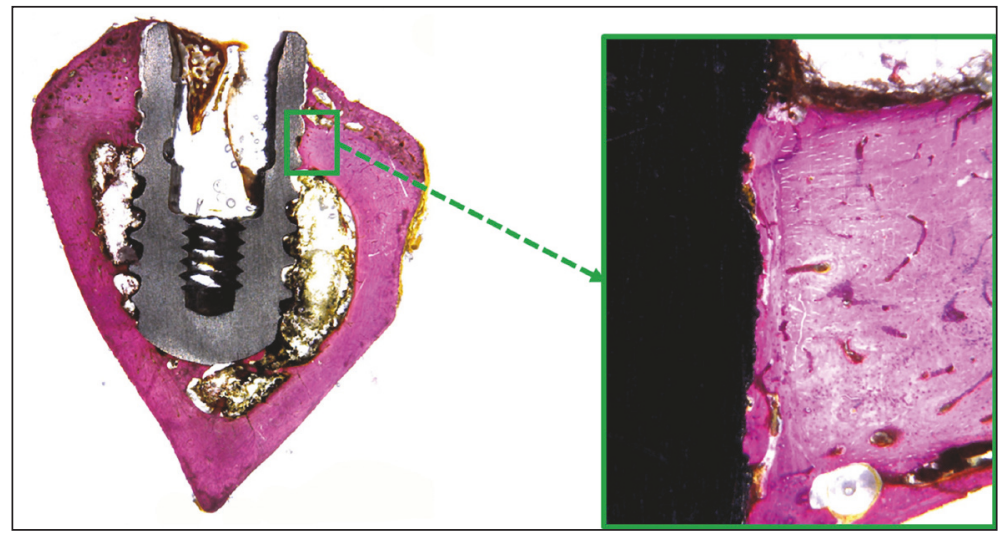

FIGURE 8: Histological pictures showing the bone healing around the implant after 6 weeks of Group 2. Magnification: 4 and 100x, respectively. Picrosirius-hematoxylin staining.

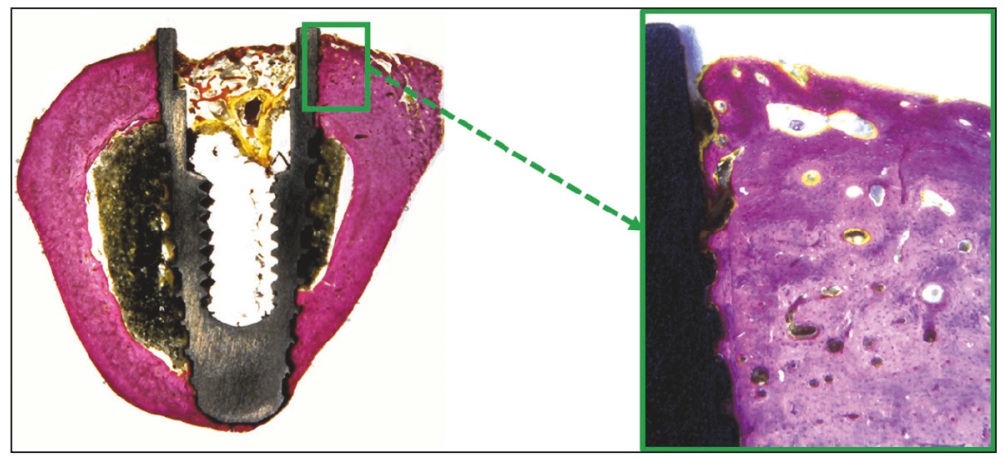

FIGURE 9: Histological pictures showing the bone healing around the implant after 6 weeks of Group 3. Magnification: 4 and 100x, respectively. Picrosirius-hematoxylin staining.

and the authors concluded that the RFA method is quite useful as a tool for clinical and noninvasive research and may help to demonstrate the behavior of implants and their relationship with peri-implant tissues, especially with respect to bone tissue. In addition, the clinical observations indicated that the final healing time was different between the study participants and the local conditions evaluated. Concerning the evolution of bone healing (osseointegration), studies have shown that there is a growing increase in SSI measurements, which was $\sim 300 \mathrm{~Hz}$ per week [42]. The measurements in present study showed statistical differences between the groups of ISQ values and baseline values (time 1). However, in time 2 (6 weeks), no differences were found. Based on an overall average of the ISQ measured among the first and 
second time, points of all groups, with this variation of RFA, increased by $14.8 \%$.

The counter-torque analyses of the implants are invasive biomechanical tests which, as previously described, can demonstrate the strength of the union between the implant and the bone and do not show several differences of the values in the same animal model [29]. Because the test requires destruction of the study specimens, in the present study the measurements were performed in triplicate and the values showed no significant difference between the groups. On the other hand, high counter-torque indices were observed in all samples of the 3 groups studied, and the results found were consistent with the results of other published studies [29] and confirm the good surface quality of the implants used in this study. In the present study, a fully computer-controlled counter-torque machine has been used, and it has been used in other studies [29], thus eliminating the possibility of any distortion in the measurements caused by the operator.

Regarding the surfaces of the implants used in the present study, basically the surfaces of Groups 1 and 2 are very similar, being both SLA surface, whose excellent osseointegration of implants is evidenced by several studies and which offers predictable long-term results $[43,44]$. Similarly, the surface presented by Group 3 implants (TiUnite) has a proven high success rate $[45,46]$. Therefore, the results presented in the this study probably had the same stimulus intensity regarding surface treatment in the 3 proposed groups.

Although the results of this technique are similar to those found in conventional implants systems, further studies on the use of single drill for osteotomy should be performed to investigate the lifetime, the wear of this drills, and the influence on the implant osseointegration.

\section{Conclusion}

Within the limitations of this animal study, the findings showed that a single drill system did not change the biomechanical and/or biological of peri-implant tissue response more than a conventional drilling sequence does while preparing implant site and may be considered as safe as the latter. The measured values in all proposed tests showed similar results for the three implant designs tested in the present study.

\section{Conflicts of Interest}

The authors declare that they have no conflicts of interest.

\section{References}

[1] D. Buser, R. Mericske-Stern, J. P. Bernard et al., "Long-term evaluation of non-submerged ITI implants. Part 1: 8-year life table analysis of a prospective multi-center study with 2359 implants," Clinical Oral Implants Research, vol. 8, no. 3, pp. 161-172, 1997.

[2] C. Mangano, F. Mangano, A. Piattelli, G. Iezzi, A. Mangano, and L. L. Colla, "Prospective clinical evaluation of 307 single-tooth morse taper-connection implants: A multicenter study," The International Journal of Oral \& Maxillofacial Implants, vol. 25, no. 2, pp. 394-400, 2010.
[3] P. I. Brånemark, U. Breine, R. Adell, B. O. Hansson, J. Lindstrom, and A. Ohlsson, "Intra-osseous anchorage of dental prostheses. I. Experimental studies," Plastic and Reconstructive Surgery, vol. 48, no. 1, pp. 97-98, 1971.

[4] T. Albrektsson, P. Brånemark, H. A. Hansson, and J. Lindstrom, "Osseointegrated titanium implants. Requirements for ensuring a long-lasting, direct bone-to-implant anchorage in man," Acta Orthopaedica, vol. 52, no. 2, pp. 155-170, 1981.

[5] T. Albrektsson, J. Brunski, and A. Wennerberg, "A requiem for the periodontal ligament' revisited," International Journal of Prosthodontics, vol. 22, no. 2, pp. 120-122, 2009.

[6] M. Esposito, J.-M. Hirsch, U. Lekholm, and P. Thomsen, "Biological factors contributing to failures of osseointegrated oral implants: (II). Etiopathogenesis," European Journal of Oral Sciences, vol. 106, no. 3, pp. 721-764, 1998.

[7] R. A. Eriksson, T. Albrektsson, and B. Magnusson, "Assessment of bone viability after heat trauma: A histological, histochemical and vital microscopic study in the rabbit," Journal of Plastic Surgery and Hand Surgery, vol. 18, no. 3, pp. 261-268, 1984.

[8] J. Lundskog, "Heat and bone tissue. An experimental investigation of the thermal properties of bone and threshold levels for thermal injury," Scandinavian Journal of Plastic and Reconstructive Surgery, vol. 9, pp. 1-80, 1972.

[9] M. Leunig and R. Hertel, "Thermal necrosis after tibial reaming for intramedullary nail fixation: A report of three cases," The Journal of Bone \& Joint Surgery (British Volume), vol. 78, no. 4, pp. 584-587, 1996.

[10] D. L. Brisman, "The Effect of Speed, Pressure, and Time on Bone Temperature during the Drilling of Implant Sites," The International Journal of Oral \& Maxillofacial Implants, vol. 11, no. 1, pp. 35-37, 1996.

[11] C. J. Kerawala, I. C. Martin, W. Allan, and E. D. Williams, “The effects of operator technique and bur design on temperature during osseous preparation for osteosynthesis self-tapping screws," Oral Surgery, Oral Medicine, Oral Pathology, Oral Radiology, and Endodontology, vol. 88, no. 2, pp. 145-150, 1999.

[12] B. H. Harris and S. S. Kohles, "Effects of mechanical and thermal fatigue on dental drill performance," The International Journal of Oral \& Maxillofacial Implants, vol. 16, pp. 819-826, 2001.

[13] N. Oliveira, F. Alaejos-Algarra, J. Mareque-Bueno, E. FerrésPadró, and F. Hernández-Alfaro, "Thermal changes and drill wear in bovine bone during implant site preparation. A comparative in vitro study: Twisted stainless steel and ceramic drills," Clinical Oral Implants Research, vol. 23, no. 8, pp. 963-969, 2012.

[14] R. Lutz, S. Srour, J. Nonhoff, T. Weisel, C. J. Damien, and K. A. Schlegel, "Biofunctionalization of titanium implants with a biomimetic active peptide (P-15) promotes early osseointegration," Clinical Oral Implants Research, vol. 21, no. 7, pp. 726-734, 2010.

[15] H. Schliephake, D. Scharnweber, S. Roesseler, M. Dard, A. Sewing, and A. Aref, "Biomimetic calcium phosphate composite coating of dental implants," The International Journal of Oral \& Maxillofacial Implants, vol. 21, no. 5, pp. 738-746, 2006.

[16] H. Schliephake, A. Aref, D. Scharnweber, S. Rößler, and A. Sewing, "Effect of modifications of dual acid-etched implant surfaces on periimplant bone formation. Part II: Calcium phosphate coatings," Clinical Oral Implants Research, vol. 20, no. 1, pp. 38-44, 2009.

[17] H. Schliephake, A. Aref, D. Scharnweber, S. Bierbaum, and A. Sewing, "Effect of modifications of dual acid-etched implant surfaces on peri-implant bone formation. Part I: organic coatings," Clinical Oral Implants Research, vol. 20, no. 1, pp. 31-37, 2009. 
[18] C. Hallgren, H. Reimers, D. Chakarov, J. Gold, and A. Wennerberg, "An in vivo study of bone response to implants topographically modified by laser micromachining," Biomaterials, vol. 24, no. 5, pp. 701-710, 2003.

[19] S. A. Gehrke, J. L. C. Guirado, R. Bettach, M. D. Fabbro, C. P.-A. Martínez, and J. A. Shibli, "Evaluation of the insertion torque, implant stability quotient and drilled hole quality for different drill design: an in vitro investigation," Clinical Oral Implants Research, 2016.

[20] A. Patel, L. F. Gil, A. Castellano et al., "Effect of simplified onestep drilling protocol on osseointegration," International Journal of Periodontics and Restorative Dentistry, vol. 36, no. 5, pp. e82-e87, 2016.

[21] G. Giro, N. Tovar, C. Marin et al., "The effect of simplifying dental implant drilling sequence on osseointegration: An experimental study in dogs," International Journal of Biomaterials, vol. 2013, Article ID 230310, 6 pages, 2013.

[22] S. A. Gehrke, "Evaluation of the cortical bone reaction around of implants using a single-use final drill: A histologic study," The Journal of Craniofacial Surgery, vol. 26, no. 5, pp. 1482-1486, 2015.

[23] R. Jimbo, G. Giro, C. Marin et al., "Simplified drilling technique does not decrease dental implant osseointegration: A preliminary report," Journal of Periodontology, vol. 84, no. 11, pp. 15991605, 2013.

[24] R. Bettach, S. Taschieri, G. Boukhris, and M. Del Fabbro, "Implant survival after preparation of the implant site using a single bur: a case series," Clinical Implant Dentistry and Related Research, vol. 17, no. 1, pp. 13-21, 2015.

[25] Basic Information on the Surgical Procedures. Straumann ${ }^{\circledR}$ Dental Implant System. In: https://www.straumann.com/.

[26] Nobel Replace ${ }^{\circledR}$ and Replace Select ${ }^{\mathrm{TM}}$ Tapered: Procedures Manual. In: https://www.nobelbiocare.com/international/en/home .html.

[27] I. C. Benington, P. A. Biagioni, P. J. Crossey, D. L. Hussey, S. Sheridan, and P.-J. Lamey, "Temperature changes in bovine mandibular bone during implant site preparation: An assessment using infra-red thermography," Journal of Dentistry, vol. 24, no. 4, pp. 263-267, 1996.

[28] G. Augustin, S. Davila, T. Udilljak, T. Staroveski, D. Brezak, and S. Babic, "Temperature changes during cortical bone drilling with a newly designed step drill and an internally cooled drill," International Orthopaedics, vol. 36, no. 7, pp. 1449-1456, 2012.

[29] S. A. Gehrke and G. W. Marin, "Biomechanical evaluation of dental implants with three different designs: Removal torque and resonance frequency analysis in rabbits," Annals of Anatomy, vol. 199, pp. 30-35, 2015.

[30] S. A. Gehrke, R. Bettach, S. Taschieri, G. Boukhris, S. Corbella, and M. Del Fabbro, "Temperature Changes in Cortical Bone after Implant Site Preparation Using a Single Bur versus Multiple Drilling Steps: An In Vitro Investigation," Clinical Implant Dentistry and Related Research, vol. 17, no. 4, pp. 700-707, 2015.

[31] L. Chong, A. Khocht, J. B. Suzuki, and J. Gaughan, "Effect of implant design on initial stability of tapered implants.", Journal of Oral Implantology, vol. 35, no. 3, pp. 130-135, 2009.

[32] J. J. McCullough and P. R. Klokkevold, "The effect of implant macro-thread design on implant stability in the early postoperative period: a randomized, controlled pilot study," Clinical Oral Implants Research, 2016.
[33] S. Huwais and E. G. Meyer, "A novel osseous densification approach in implant osteotomy preparation to increase biomechanical primary stability, bone mineral density, and bone-toimplant contact," The International Journal of Oral \& Maxillofacial Implants, vol. 32, no. 1, pp. 27-36, 2017.

[34] M. Textor, C. Sittig, V. Frauchiger, S. Tosatti, and D. M. Brunette, "Properties and Biological Significance of Natural Oxide Films on Titanium and Its Alloys," in Titanium in Medicine, D. M. Brunette, P. Tengvall, M. Textor, and P. Thomsen, Eds., Engineering Materials, pp. 171-230, Springer Berlin Heidelberg, Berlin, Germany, 2001.

[35] H. J. Oh, U. M. Wikesjö, H.-S. Kang, Y. Ku, T.-G. Eom, and K.T. Koo, "Effect of implant drill characteristics on heat generation in osteotomy sites: A pilot study," Clinical Oral Implants Research, vol. 22, no. 7, pp. 722-726, 2011.

[36] G. Augustin, T. Zigman, S. Davila et al., "Cortical bone drilling and thermal osteonecrosis," Clinical Biomechanics, vol. 27, no. 4, pp. 313-325, 2012.

[37] N. Meredith, "Assessment of implant stability as a prognostic determinant," International Journal of Prosthodontics, vol. 11, no. 5, pp. 491-501, 1998.

[38] J. Steigenga, K. Al-Shammari, C. Misch, F. H. Nociti Jr., and H.L. Wang, "Effects of implant thread geometry on percentage of osseointegration and resistance to reverse torque in the tibia of rabbits," Journal of Periodontology, vol. 75, no. 9, pp. 1233-1241, 2004.

[39] U. T. Da Silva Neto, J. C. Joly, and S. A. Gehrke, "Clinical analysis of the stability of dental implants after preparation of the site by conventional drilling or piezosurgery," British Journal of Oral and Maxillofacial Surgery, vol. 52, no. 2, pp. 149-153, 2014.

[40] N. Meredith, D. Alleyne, and P. Cawley, "Quantitative determination of the stability of the implant-tissue interface using resonance frequency analysis," Clinical Oral Implants Research, vol. 7, no. 3, pp. 261-267, 1996.

[41] S. A. Gehrke and U. T. da Silva Neto, "Does the Time of Osseointegration in the Maxilla and Mandible Differ?" The Journal of Craniofacial Surgery, vol. 25, no. 6, pp. 2117-2120, 2014.

[42] N. Meredith, B. Friberg, L. Sennerby, and C. Aparicio, "Relationship between contact time measurements and PTV values when using the Periotest to measure implant stability," The International Journal of Prosthodontics, vol. 11, no. 3, pp. 269275, 1998.

[43] F. J. J. van Velzen, R. Ofec, E. A. J. M. Schulten, and C. M. ten Bruggenkate, "10-year survival rate and the incidence of periimplant disease of 374 titanium dental implants with a SLA surface: A prospective cohort study in 177 fully and partially edentulous patients," Clinical Oral Implants Research, vol. 26, no. 10, pp. 1121-1128, 2015.

[44] D. Buser, S. F. M. Janner, J.-G. Wittneben, U. Brägger, C. A. Ramseier, and G. E. Salvi, "10-year survival and success rates of 511 titanium implants with a sandblasted and acid-etched surface: a retrospective study in 303 partially edentulous patients," Clinical Implant Dentistry and Related Research, vol. 14, no. 6, pp. 839-851, 2012.

[45] M. Degidi, D. Nardi, and A. Piattelli, "10-year follow-up of immediately loaded implants with TiUnite porous anodized surface," Clinical Implant Dentistry and Related Research, vol. 14, no. 6, pp. 828-838, 2012.

[46] M. Degidi, V. Perrotti, and A. Piattelli, "Immediately loaded titanium implants with a porous anodized surface with at least 36 months of follow-up," Clinical Implant Dentistry and Related Research, vol. 8, no. 4, pp. 169-177, 2006. 


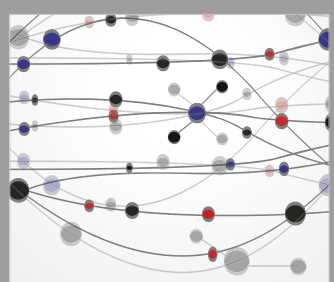

The Scientific World Journal
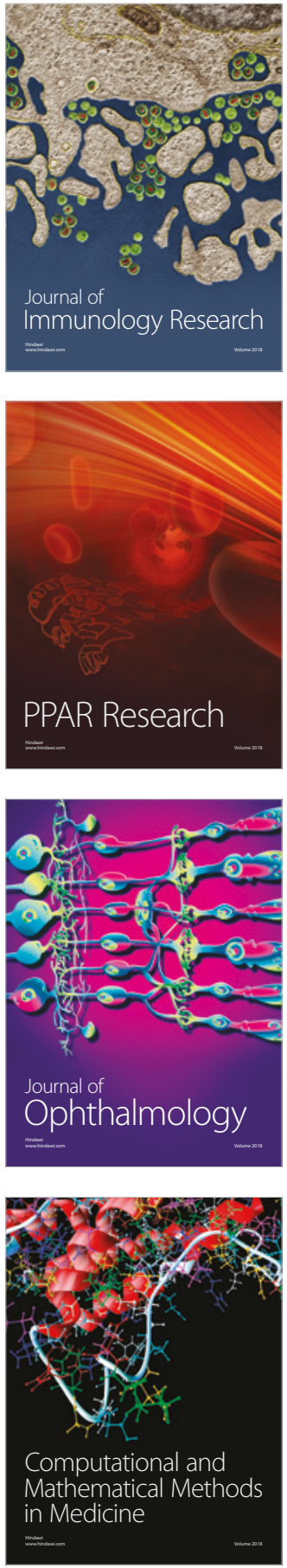

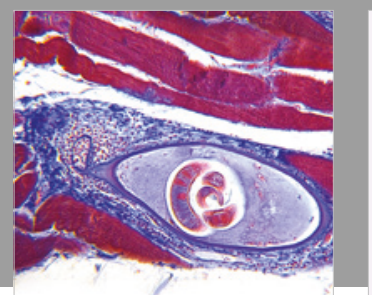

Gastroenterology Research and Practice

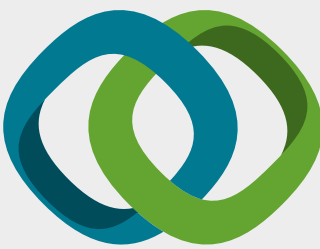

\section{Hindawi}

Submit your manuscripts at

www.hindawi.com
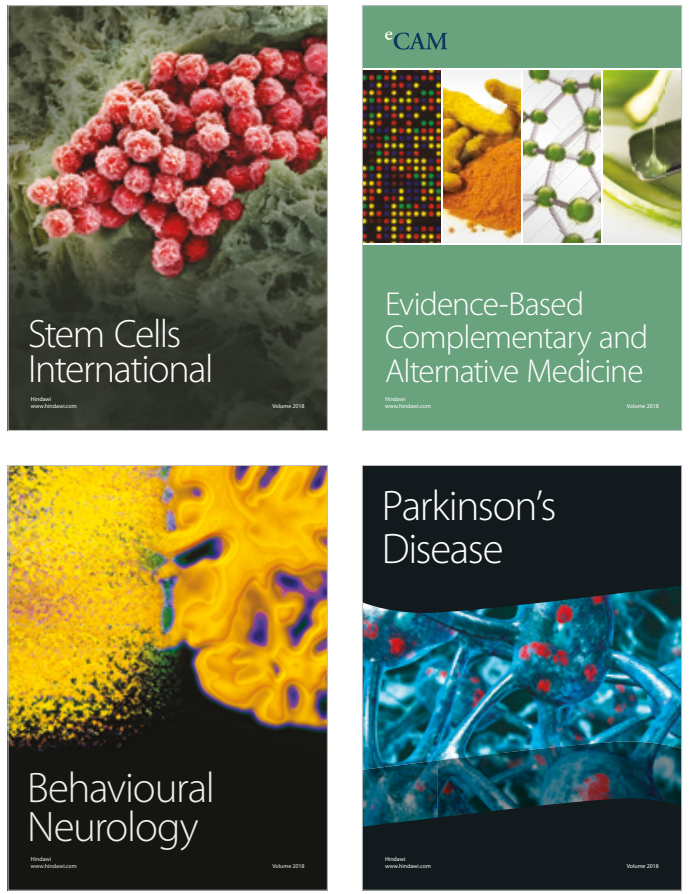

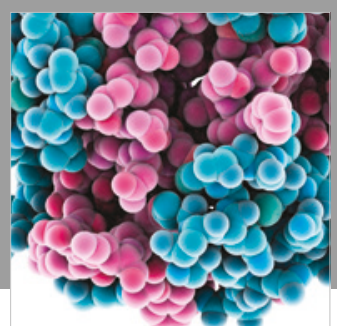

ournal of

Diabetes Research

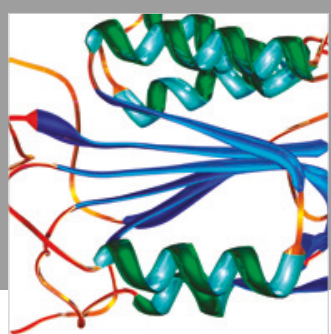

Disease Markers
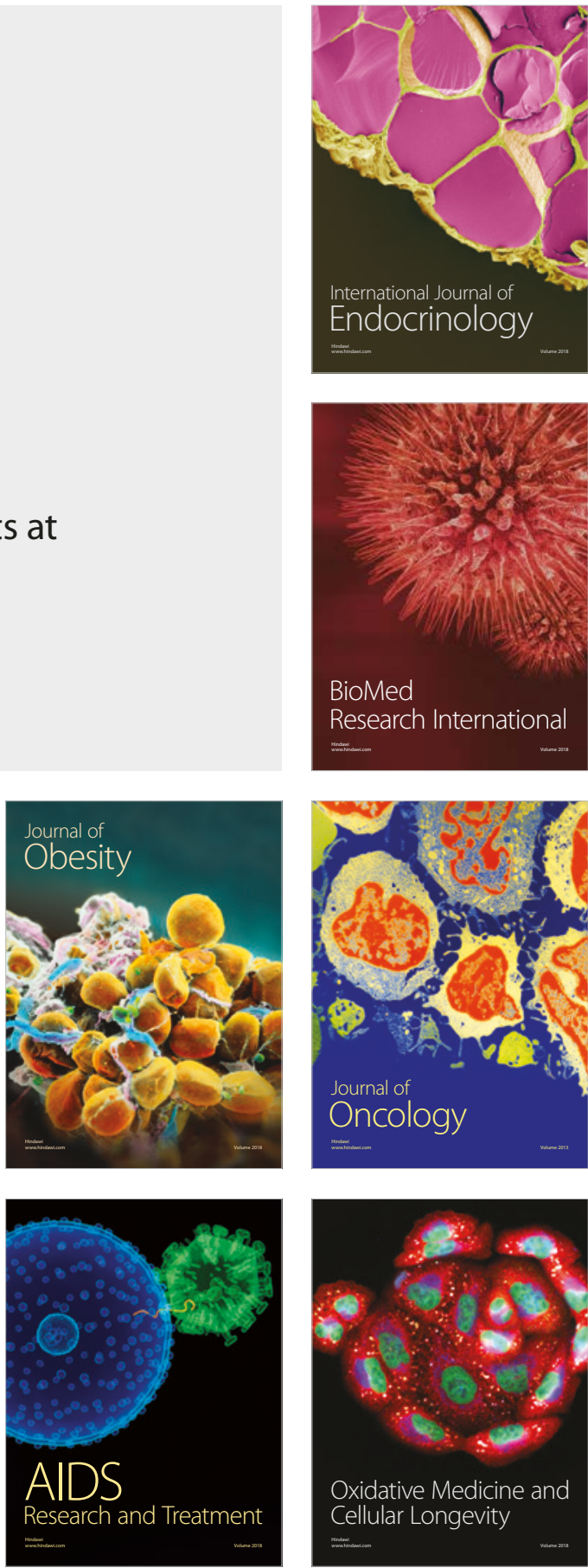